\title{
Estudio de los mecanismos de activación de la esfalerita con Cu(II) y $\mathrm{Pb}(\mathrm{II})^{(\bullet)}$
}

\author{
G. I. Dávila Pulido* y A. Uribe Salas*
}

\begin{abstract}
Resumen
Este artículo presenta los resultados de un estudio experimental sobre la activación de esfalerita ( $\mathrm{ZnS}$ ) con Cu(II) y $\mathrm{Pb}(\mathrm{II})$, cuyo objetivo principal consistió en investigar los mecanismos de activación y en evaluar la magnitud relativa de la hidrofobización alcanzada con ambas especies químicas. La hidrofobicidad que la superficie mineral adquiere como resultado de la interacción con los activadores y colectores tipo xantato (ditiocarbonatos alquílicos, R-O-CS ${ }_{2}^{-}$), se caracteriza mediante la técnica del ángulo de contacto. Los resultados muestran que el $\mathrm{Cu}(\mathrm{II})$ es intercambiado por el Zn de las capas exteriores del cristal, promoviendo la oxidación de sulfuro $\left(\mathrm{S}^{2-}\right)$ para producir una mezcla de CuS, $\mathrm{Cu}_{2} \mathrm{~S}_{\text {y }} \mathrm{S}^{\circ}$, de naturaleza hidrofóbica. La interacción posterior con el xantato, hace que la hidrofobicidad de la superficie se incremente. Por su parte, la activación con $\mathrm{Pb}(\mathrm{II})$ se debe a la formación de una capa de $\mathrm{PbS}$, la cual reacciona espontáneamente con el xantato para producir especies hidrofóbicas (e.g., $\mathrm{PbX}_{2}$ ). Se observa que la hidrofobización de la esfalerita acondicionada con $\mathrm{Pb}(\mathrm{II})$ se favorece en atmósferas de aire, en comparación con la obtenida en atmósferas de nitrógeno. Se concluye que la hidrofobización alcanzada de manera inadvertida con el $\mathrm{Pb}(\mathrm{II})$, puede llegar a ser del mismo orden de magnitud que la hidrofobización inducida deliberadamente mediante la activación con cobre.
\end{abstract}

\section{Contact angle study on the activation mechanisms of sphalerite with $\mathrm{Cu}$ (II) and $\mathrm{Pb}$ (II)}

\begin{abstract}
This article presents results of an experimental study on the sphalerite activation with $\mathrm{Cu}(\mathrm{II})$ and $\mathrm{Pb}(\mathrm{II})$, whose main objective was to investigate the activation mechanisms and to evaluate the magnitude of the hydrophobization achieved with both chemical species. The hydrophobicity acquired by the mineral due to the interaction with the activator and collector (sodium isopropyl xanthate) is characterized making use of the contact angle technique. The results show that $\mathrm{Cu}(\mathrm{II})$ replaces the $\mathrm{Zn}$ of the external layers of the mineral, promoting the sulfide $\left(\mathrm{S}^{2-}\right)$ oxidation to produce a mixture of $\mathrm{CuS}, \mathrm{Cu}_{2} \mathrm{~S}$ and $\mathrm{S}^{\circ}$, of hydrophobic nature. The subsequent interaction with xanthate increases the hydrophobicity of the mineral surface. In turn, $\mathrm{Pb}(\mathrm{II})$ activation of sphalerite is due to the formation of a PbS layer that reacts with xanthate to produce hydrophobic species (e.g., $\mathrm{PbX}{ }_{2}$ ). It is also observed that the hydrophobicity of sphalerite activated with $\mathrm{Pb}$ (II) is favored under air atmospheres, as compared to that obtained under nitrogen atmospheres. It is concluded that the hydrophobicity achieved by lead activation may be of the same order of magnitude to that deliverately induced by copper activation.
\end{abstract}

Keywords $\quad$ Sphalerite; Copper activation; Lead activation; Flotation; Contact angle.

\section{INTRODUCCIÓN}

Los minerales sulfurosos de zinc tales como la esfalerita $(\mathrm{ZnS})$ y la marmatita $((\mathrm{ZnFe}) \mathrm{S})$, en general no se hidrofobizan de manera importante cuando se ponen en contacto con colectores tipo xantato (i.e., ditiocarbonatos alquílicos) de cadenas alquílicas cortas, por lo que se hace necesario su activación con cobre con el propósito de formar una capa de sulfuro de cobre sobre la esfalerita, el cual sí posee afinidad por dichos colectores. El proceso mediante el cual los átomos de cobre son incorporados a la superficie de la esfalerita se denomina "activación con cobre" y se lleva a cabo deliberadamente en aquellos minerales en los que la esfalerita es la especie valiosa; sin embargo, la esfalerita también puede ser activada de manera "inadvertida" debido a los iones de cobre, plomo, plata, etc., provenientes del

\footnotetext{
(•) Trabajo recibido el día 27 de Agosto de 2010 y aceptado en su forma final el día 14 de Abril de 2011

* CINVESTAV-IPN Unidad Saltillo, Carretera Saltillo-Monterrey km 13, Col. Molinos del Rey, Ramos Arizpe, Coahuila, 25900 México. gloria.davilapulido@cinvestav.edu.mx.
} 
propio sistema (i.e., oxidación de los minerales, uso de agua reciclada, etc.). En este último caso, las especies metálicas tienen un efecto perjudicial en la separación debido a que pueden provocar la activación de la esfalerita en el circuito de plomo/cobre, en el que se busca flotar las especies de plomo y cobre y dejar a la esfalerita en las colas. Asimismo, los iones pueden emigrar de un mineral a otro, reduciendo las diferencias inherentes que existen entre estos, dando como resultado una pérdida de la selectividad $^{[1]}$. Como se mencionó arriba, los colectores más empleados en esta aplicación son los ditiocarbonatos alquílicos, mejor conocidos como xantatos, su nombre comercial, debido a su efectividad y bajo costo.

El presente trabajo hace uso de la técnica del ángulo de contacto, debido a que este representa una medida directa de la hidrofobicidad de la superficie mineral ${ }^{[2]}$. Recientemente, CSIRO (Commonwealth Scientific and Industrial Research Organization), Australia, ha desarrollado un modelo fluidinámico computacional para celdas de flotación agitadas mecánicamente, en el que el ángulo de contacto es una de los variables de entrada de mayor importancia, que determinan la precisión y aplicabilidad del modelo ${ }^{[3}$ y 4$]$. La activación inadvertida de $\mathrm{ZnS}$ con el $\mathrm{Pb}(\mathrm{II})$ proveniente de la oxidación natural de la galena durante la molienda, el acondicionamiento y la flotación, puede reducir la selectividad de la separación, afectando adversamente el grado del concentrado de plomo y la recuperación global del zinc, debido al zinc recuperado (i.e., perdido) en el concentrado de plomo. El presente trabajo aborda el estudio experimental de los mecanismos de activación de esfalerita con $\mathrm{Cu}(\mathrm{II})$ y $\mathrm{Pb}(\mathrm{II})$, así como la medi- ción de la hidrofobicidad relativa alcanzada con ambas especies.

\section{METODOLOGÍA EXPERIMENTAL}

La metodología experimental empleada consistió en someter al cristal de esfalerita a un acondicionamiento químico de activación con $\mathrm{Cu}$ (II) o $\mathrm{Pb}(\mathrm{II})$ y de acondicionamiento con xantato isopropílico de sodio (XINa), bajo condiciones de $\mathrm{pH}$ y tiempos de acondicionamiento similares a los empleados en los circuitos de flotación convencionales (Tabla I y II). La fuerza iónica de todas las soluciones empleadas se mantuvo constante $\left(10^{-3} \mathrm{M} \mathrm{NaNO}_{3}\right)$ con el objeto de mantener constante el coeficiente de actividad de las especies acuosas. Al final de las etapas de acondicionamiento, se midió el ángulo de contacto que el mineral hace con una burbuja de aire, el cual se sabe que es una buena medida de la flotabilidad del mineral.

\subsection{Caracterización del mineral}

Las mediciones de ángulo de contacto se realizaron con cristales de esfalerita procedentes del distrito minero de Bismark (Mpio. de La Ascensión, Chih., México), cuya composición química fue determinada mediante análisis elemental (59,1\% Zn, 29,1\% S, 5,7 \% Fe en solución sólida y alrededor de $6 \%$ insolubles), mientras que su composición mineralógica fue determinada mediante difracción de rayos X. El patrón de difracción correspondiente a la carta

Tabla I. Condiciones experimentales empleadas durante la activación de esfalerita con Cu(II)

Table I. Experimental conditions for the activation of sphalerite with $\mathrm{Cu}(\mathrm{II})$

\begin{tabular}{|c|c|c|c|c|c|c|c|}
\hline & \multicolumn{3}{|c|}{$\begin{array}{c}\text { Etapa I de } \\
\text { acondicionamiento }\end{array}$} & \multicolumn{4}{|c|}{$\begin{array}{c}\text { Etapa II de } \\
\text { acondicionamiento }\end{array}$} \\
\hline & $\begin{array}{c}{[\mathrm{Cu}]} \\
(\mathrm{ppm})\end{array}$ & pH & $\begin{array}{l}\text { tiempo } \\
\text { (min) }\end{array}$ & $\begin{array}{l}\mathrm{NaNO}_{3} \\
\text { (M) }\end{array}$ & $\begin{array}{l}\text { XINa } \\
\text { (M) }\end{array}$ & $\mathrm{pH}$ & $\begin{array}{l}\text { tiempo } \\
\text { (min) }\end{array}$ \\
\hline Activación de ZnS con $\mathrm{Cu}^{2+}$ & $0-50$ & 5,4 & 5 y 15 & $10^{-3}$ & - & 10 & 5 \\
\hline Activación de $\mathrm{ZnS}$ con $\mathrm{Cu}(\mathrm{OH})_{2}$ & $0-50$ & 9,0 & 15 & $10^{-3}$ & - & 10 & 5 \\
\hline $\begin{array}{l}\text { Efecto del pH de flotación de la } \\
\text { esfalerita activada con } \mathrm{Cu}^{2+}\end{array}$ & 50 & 5,4 & 15 & $10^{-3}$ & - & $5-10$ & 5 \\
\hline $\begin{array}{l}\text { Efecto de la interacción del XINa } \\
\text { con la esfalerita activada por las } \\
\text { especies } \mathrm{Cu}^{2+} \text { y } \mathrm{Cu}(\mathrm{OH})_{2}\end{array}$ & $0-50$ & 5,4 y 9,0 & 15 & $10^{-3}$ & $10^{-4}$ & 10 & 5 \\
\hline
\end{tabular}

Nota: Las soluciones utilizadas en la Etapa I de acondicionamiento se prepararon utilizando una fuerza iónica de $10^{-3} \mathrm{M}_{\text {de }} \mathrm{NaNO}_{3}$. 
ESTUDIO DE LOS MECANISMOS DE ACTIVACIÓN DE LA ESFALERITA CON CU(II) Y PB(II) CONTACT ANGLE STUDY ON THE ACTIVATION MECHANISMS OF SPHALERITE WITH CU (II) AND PB (II)

Tabla II. Condiciones experimentales empleadas en los experimentos de activación de esfalerita con $\mathrm{Pb}(\mathrm{II})$

Table II. Experimental conditions used in the experiments of sphalerite activation with $\mathrm{Pb}(\mathrm{II})$

\begin{tabular}{|c|c|c|c|c|c|c|c|}
\hline & \multicolumn{3}{|c|}{$\begin{array}{l}\text { Etapa I de } \\
\text { acondicionamiento }\end{array}$} & \multicolumn{4}{|c|}{$\begin{array}{l}\text { Etapa II de } \\
\text { acondicionamiento }\end{array}$} \\
\hline & $\begin{array}{c}{[\mathrm{Pb}]} \\
(\mathrm{ppm})\end{array}$ & pH & $\begin{array}{l}\text { tiempo } \\
\text { (min) }\end{array}$ & $\begin{array}{l}\mathrm{NaNO}_{3} \\
\text { (M) }\end{array}$ & $\begin{array}{c}\mathrm{XINa} \\
(\mathrm{M})\end{array}$ & pH & $\begin{array}{c}\text { tiempo } \\
\text { (min) }\end{array}$ \\
\hline $\begin{array}{l}\text { Activación de } \mathrm{ZnS} \text { con } \mathrm{Pb}(\mathrm{II}) \text { en } \\
\text { ausencia y presencia de oxígeno(a) }\end{array}$ & 200 & 9,0 & 15 & $10^{-3}$ & - & 9,0 & 5 \\
\hline $\begin{array}{l}\text { Efecto de la interacción del XINa } \\
\text { con la esfalerita activada con } \mathrm{Pb}(\mathrm{II})\end{array}$ & $0-250$ & 9,0 & 15 & $10^{-3}$ & $10^{-4}$ & 9,0 & 5 \\
\hline $\begin{array}{l}\text { Efecto del pH sobre la activación } \\
\text { de la esfalerita con } \mathrm{Pb}(\mathrm{II})\end{array}$ & 150 & 9,0 & 15 & $10^{-3}$ & $10^{-4}$ & 9,0 & 5 \\
\hline
\end{tabular}

(a) Los resultados de estos experimentos se presentan en la Figura 7

Nota: Las soluciones utilizadas en la Etapa I de acondicionamiento se prepararon utilizando una fuerza iónica de $10^{-3} \mathrm{M}$ de $\mathrm{NaNO}_{3}$.

01-077-2100, indica que solamente se cuenta con una fase primaria correspondiente a la esfalerita ( $\mathrm{ZnS})$, por lo que se puede considerar que los cristales son prácticamente puros.

\subsection{Técnica de medición de ángulo de contacto}

En general las técnicas de medición de ángulo de contacto se dividen en dos categorías: Superficies planas o ideales y superficies no ideales. En el presente trabajo se utilizaron superficies planas; el método más común para la caracterización de este tipo de superficies se basa en el uso de dispositivos ópticos de baja magnificación para observar la imagen de la gota o burbuja de aire sobre la superficie del cristal mineral.

Los experimentos de ángulo de contacto se llevaron a cabo utilizando cristales minerales, los cuales fueron seleccionados y montados en resina metalográfica; las probetas así obtenidas se devastaron y pulieron con lijas de carburo de silicio (No. 80 a 800 ) y con agua desionizada y desoxigenada, de acuerdo a la técnica propuesta en la literatura ${ }^{[5]} \mathrm{y}$ reportada por $\mathrm{Chau}^{[3]}$; es importante mencionar que el procedimiento de pulido fino de los cristales se lleva a acabo previo a cada prueba, con el fin de renovar la superficie del cristal. Una vez que la etapa de preparación de la muestra mineral concluye, se lleva a cabo el acondicionamiento con los tiempos y condiciones químicas de interés (e.g., $\mathrm{pH}$, tiempo de acondicionamiento, concentración de cobre, con- centración de XINa), los cuales simulan aproximadamente las condiciones de los circuitos industriales de flotación de esfalerita (Tabla I y II); en todas las etapas de acondicionamiento la temperatura se mantuvo constante en $30^{\circ} \mathrm{C}$. Concluido el proceso de acondicionamiento, la probeta es transferida a un contenedor de acrílico con una solución acuosa de fuerza iónica constante $\left(10^{-3} \mathrm{M} \mathrm{NaNO}_{3}\right)$ y $\mathrm{pH} 10$, en donde se lleva a cabo el contacto entre el cristal y una burbuja de aire con un diámetro aproximado de $1 \mathrm{~mm}$, producida con una jeringa digital Hamilton, e inmediatamente se procede a la toma de las fotografías del contacto entre las 3 fases, las cuales fueron procesadas con la ayuda de un programa de análisis de imágenes (Image - Pro 5.1). La toma de fotografías se llevó a cabo con una cámara digital equipada con un lente macro $5 \mathrm{X}$ de $65 \mathrm{~mm}$ y un campo visual de $4,33 \times 2,88 \mathrm{~mm}$. Los experimentos se realizaron por triplicado por lo que se reporta el valor promedio del ángulo de contacto, así como las barras de error que delimitan el intervalo de confianza del $99 \%$ de una distribución t-student.

El ángulo de contacto entre las fases mineral-solución acuosa-aire fue obtenido de la derivada de la ecuación del círculo que mejor describe la burbuja adherida al cristal de esfalerita, calculada en el punto de contacto de las tres fases ("X" en la Fig. 1), como se describe a continuación:

Utilizando la ecuación del círculo:

$$
x^{2}+(y-k)^{2}=R^{2} \quad 0 \leq k \leq R
$$




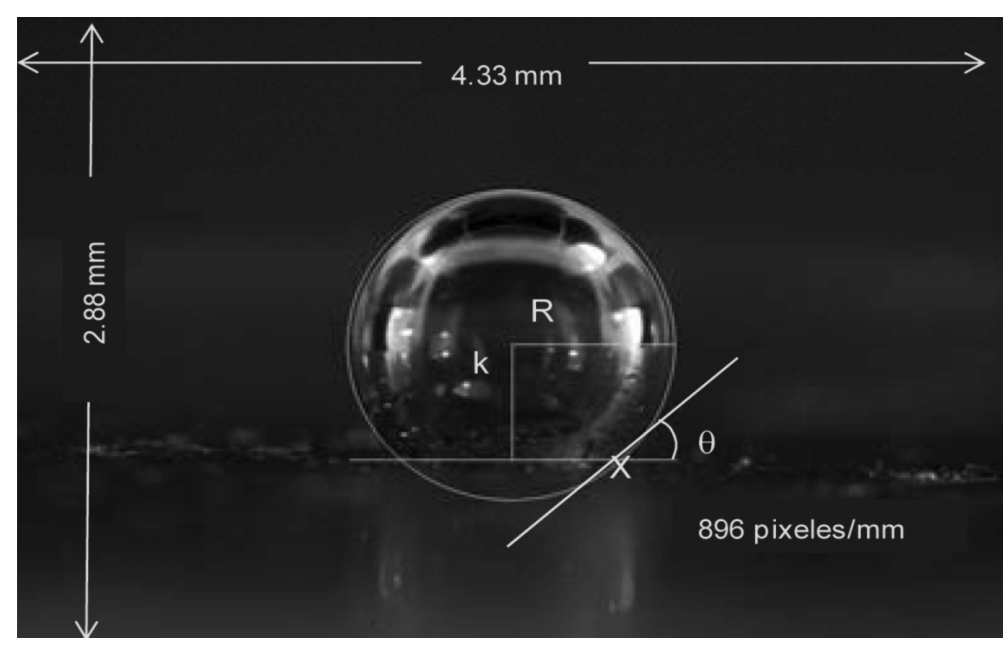

Figura 1. Fotografía que ilustra la metodología empleada para estimar la pendiente del círculo en el punto de contacto de las tres fases.

Figure 1. Photograph that illustrates the methodology used to estimate the slope to the circle at the point of contact of the three phases.

El punto de intersección entre el círculo y el eje $\mathrm{x}$ (punto "X" en la figura), se estima resolviendo la ecuación para $\mathrm{y}=0$ :

$$
x=\sqrt{R^{2}-k^{2}}
$$

La pendiente del círculo en cualquier punto $(\mathrm{x}, \mathrm{y})$, se obtiene de la derivada total de la ecuación respecto de $\mathrm{x}$ :

$$
\begin{gathered}
2 x+2 y \frac{d y}{d x}-2 k \frac{d y}{d x}=0 \\
\operatorname{tg} \theta=\frac{d y}{d x}=\frac{-2 x}{2 y-2 k}
\end{gathered}
$$

La pendiente (i.e., $\operatorname{tg} \theta$ ) se obtiene midiendo $\mathrm{k} y$ $\mathrm{R}$ en la fotografía del equilibrio de fuerzas de las 3 fases y calculando la pendiente en el punto de coordenadas $\left(\sqrt{R^{2}-k^{2}}, 0\right)$. Finalmente, del valor de la pendiente se obtiene el ángulo de contacto $\theta$.

\section{RESULTADOS Y DISCUSIÓN}

Las variables naturales del proceso de activación de la esfalerita con $\mathrm{Cu}$ (II) son la concentración de $\mathrm{Cu}(\mathrm{II})$, el $\mathrm{pH}$ y el tiempo de acondicionamiento. Con el fin de evaluar la importancia relativa y la contribución efectiva de estas variables al proceso de activación y al desarrollo de las propiedades hidrofóbicas del mineral, se diseñaron series de experimentos en las que el efecto de éstas fue evaluado individualmente.

\subsection{Activación de esfalerita con $\mathrm{Cu}(\mathrm{II})$}

La activación de la esfalerita con cobre se puede llevar a cabo mediante dos diferentes mecanismos, los cuales dependerán de la especie presente en la solución (e.g., $\mathrm{Cu}^{2+}$ y $\mathrm{Cu}(\mathrm{OH})_{2}$ ). Como se observa en el diagrama de distribución de especies del sistema $\mathrm{Cu}-\mathrm{H}_{2} \mathrm{O}$ a $25^{\circ} \mathrm{C}$ (Fig. 2), la especie responsable de la activación a $\mathrm{pH}$ ligeramente ácido $(\mathrm{pH}=5,4)$ es el $\mathrm{Cu}^{2+}$ ya que es la especie predominante a este valor de $\mathrm{pH}$, mientras que a $\mathrm{pH} 9$ la especie predominante es el $\mathrm{Cu}(\mathrm{OH})_{2}$, la cual será la responsable de la activación, de acuerdo al mecanismo de precipitación superficial. Por otro lado, Debido a que la cinética de la activación de la esfalerita con $\mathrm{Cu}(\mathrm{II})$ depende directamente de la actividad del cobre en solución ${ }^{[6]}$, aquellas condiciones donde el catión $\mathrm{Cu}^{2+}$ sea la especie predominante del sistema $\mathrm{Cu}(\mathrm{II})-\mathrm{H}_{2} \mathrm{O}(\mathrm{pH} 5,4)$, favorecerán la activación y por lo tanto, la hidrofobización del mineral. En consecuencia, también se espera que la predominancia del $\mathrm{Cu}(\mathrm{OH})_{2}$ precipitado (e.g., $\mathrm{pH}$ 9), de lugar a menores velocidades de activación y, por lo tanto, al desarrollo de una menor hidrofobicidad. 


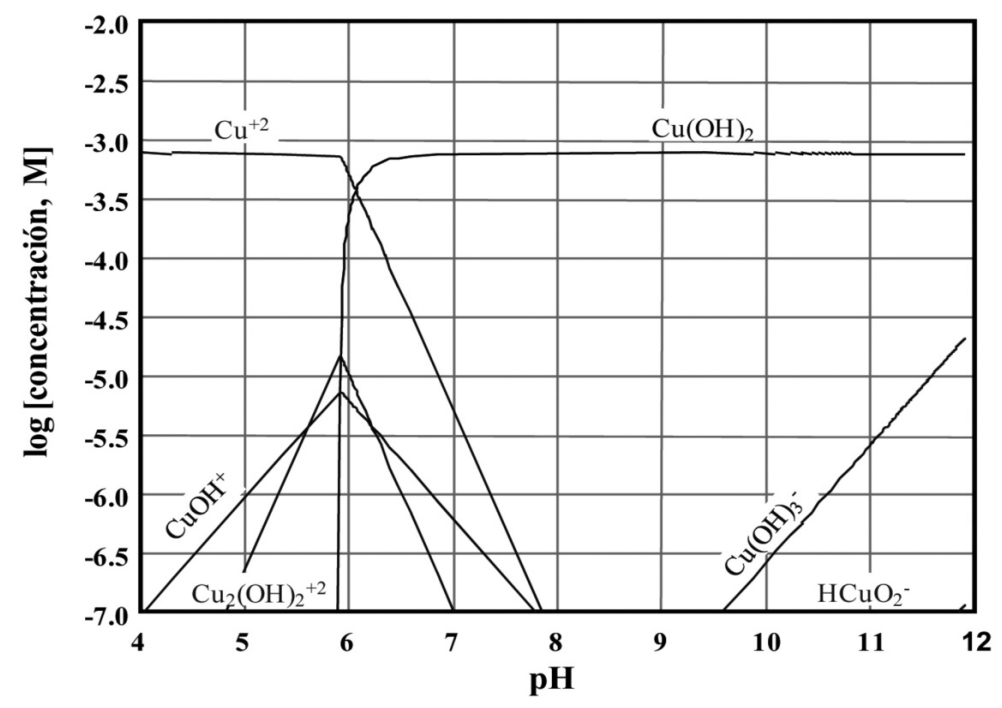

Figura 2. Diagrama de distribución de especies del sistema $\mathrm{Cu}-\mathrm{H}_{2} \mathrm{O}$ a $25^{\circ} \mathrm{C}$ y una concentración de $\mathrm{Cu}=50 \mathrm{mg} / \mathrm{L}$ (7.86 $\left.\times 10^{-4} \mathrm{M}\right)$, HSC Chemistry v. 6.1 .

Figure 2. Species distribution diagram of the $\mathrm{Cu}-\mathrm{H}_{2} \mathrm{O}$ system at $25^{\circ} \mathrm{C}$ and $[\mathrm{Cu}]=50 \mathrm{mg} / \mathrm{L}\left(7.86 \times 10^{-4} \mathrm{M}\right), \mathrm{HSC}$ Chemistry v. 6.1.

\subsubsection{Activación de esfalerita con $\mathrm{Cu}^{2+}$ (intercambio iónico).}

En la figura 3 se presentan los resultados de la activación de esfalerita en función de la concentración de $\mathrm{Cu}^{2+}$ a pH 5,4 y dos diferentes tiempo de acondicionamiento, en la cual se observa que el ángulo de contacto aumenta con la concentración de $\mathrm{Cu}(\mathrm{II})$, debido a la formación de especies hidrofóbicas en la superficie mineral (e.g., azufre elemental, sulfuro rico en azufre o bien un polisulfuro), de acuerdo al mecanismo de activación descrito por la ecuación (5) [7], donde, además del intercambio de iones, la adsorción e incorporación de $\mathrm{Cu}(\mathrm{II})$ en la red cristalina de la esfalerita provoca una reacción de oxidaciónreducción en la que el $\mathrm{Cu}$ (II) oxida al sulfuro del mineral, reduciéndose a $\mathrm{Cu}(\mathrm{I})$, dando como productos finales a la covelita $(\mathrm{CuS})$, la calcocita $\left(\mathrm{Cu}_{2} \mathrm{~S}\right)$ y azufre elemental, de naturaleza hidrofóbica; la proporción de estas especies en la superficie mineral dependerá del tiempo de reacción.

$$
Z n S+C u(I) \rightarrow C u S+Z n(I I) \rightarrow 1 / 2 C u_{2} S+1 / 2 S+Z n(I I)
$$

La ecuación de esta reacción es válida solo en medios ácidos o ligeramente ácidos, donde $\mathrm{el} \mathrm{Cu}^{2+}$ y el $\mathrm{Zn}^{2+}$ son las especies estables de los sistemas $\mathrm{Cu}-\mathrm{H}_{2} \mathrm{O}$ y $\mathrm{Zn}-\mathrm{H}_{2} \mathrm{O}$, respectivamente.
El proceso de activación es controlado por una reacción de intercambio iónico entre el $\mathrm{Cu}^{2+}$ y el $\mathrm{Zn}^{2+}$, el cual se lleva a cabo mediante la sustracción de un mol de $\mathrm{Cu}^{2+}$ (debido a su incorporación dentro de la red de la esfalerita) por cada mol de $\mathrm{Zn}^{2+}$ que es liberado del mineral, de acuerdo al modelo descrito por la ecuación (6) [6].

$$
\mathrm{MeS}+\mathrm{M}^{2+} \rightarrow \mathrm{MS}+\mathrm{Me}^{2+}
$$

Adicionalmente, en la figura 3 se observa que el tiempo de acondicionamiento juega un papel muy importante en el desarrollo del ángulo de contacto, ya que para concentraciones de $\mathrm{Cu}$ de $50 \mathrm{ppm}$ y tiempos de acondicionamiento de 5 y $15 \mathrm{~min}$, se obtienen ángulos de contacto de $25,7^{\circ}$ y $34,1^{\circ}$, respectivamente. Este aumento en la hidrofobicidad se debe a que a medida que aumenta el tiempo de reacción, es decir, el tiempo de acondicionamiento, también se incrementa la cantidad de especies hidrofóbicas en la superficie mineral.

\subsubsection{Activación de esfalerita con $\mathrm{Cu}(\mathrm{OH})_{2}$}

Con el objetivo de evaluar la contribución del $\mathrm{pH}$ al que se lleva a cabo la activación, se diseñaron experimentos a $\mathrm{pH} 9$ dado que a este valor de $\mathrm{pH}$ el 


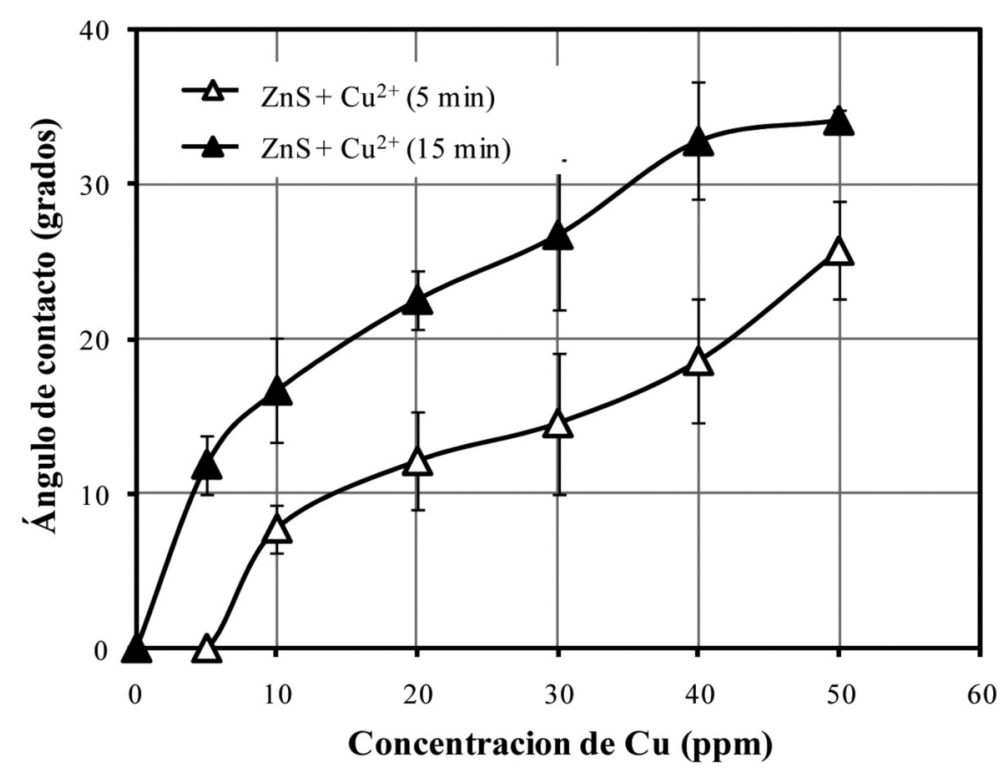

Figura 3. Efecto de la concentración de $\mathrm{Cu}^{2+}$ y del tiempo de acondicionamiento sobre el ángulo de contacto de la esfalerita. Las pruebas se realizaron a $30^{\circ} \mathrm{C}$, pH natural $(5,4)$ y una fuerza iónica de $10^{-3} \mathrm{M}$ de $\mathrm{NaNO}_{3}$; la medición de ángulo de contacto se llevó a cabo a pH 10.

Figure 3. Effect of $\mathrm{Cu}^{2+}$ concentration and conditioning time on the sphalerite contact angle. The tests were performed at $30{ }^{\circ} \mathrm{C}$, natural $\mathrm{pH}$ (5.4) and ionic strength of $10^{-3} \mathrm{M} \mathrm{NaNO}_{3}$; the contact angle measurement was carried out at $\mathrm{pH} 10$.

mecanismo que opera es el de precipitación superficial; los resultados obtenidos se presentan en la figura 4, en la que se observan ángulos de contacto de $0^{\circ}$ para concentraciones de cobre menores o iguales a $20 \mathrm{ppm}$, y un incremento lineal de éste para concentraciones de $\mathrm{Cu}$ (II) mayores a $20 \mathrm{ppm}$, hasta alcanzar valores de $28^{\circ}$ para $50 \mathrm{ppm}$ de $\mathrm{Cu}$ (II). Estos resultados evidencian que la cinética de reacción entre el $\mathrm{Cu}(\mathrm{OH})_{2}$ y la esfalerita es lenta debido a las bajas actividades de las especies de $\mathrm{Cu}(\mathrm{II})$ en solución, principalmente $\mathrm{Cu}^{2+} \mathrm{y} \mathrm{CuOH}^{+}$, quienes son finalmente las que se adsorben sobre la superficie del mineral y sustituyen al $\mathrm{Zn}^{2+}$, que es entonces liberado lentamente de la red cristalina, permaneciendo sobre la superficie del mineral en forma de hidróxido. Este mecanismo fue propuesto por Prestidge y col. ${ }^{\left[{ }^{[}\right]}$ y reportado por Chandra y Gerson ${ }^{[7]}$, y ocurre de acuerdo a las siguientes reacciones:

$$
n Z n S+x C u(O H)_{2(\text { precipitado) }} \rightarrow(\mathrm{ZnS})_{n} \cdot x \mathrm{Cu}(\mathrm{OH})_{2(\text { superficie })}
$$

El Cu(II) del hidróxido se puede entonces intercambiar por el $\mathrm{Zn}$ (II) del sulfuro

$$
(\mathrm{ZnS})_{n} \cdot x \mathrm{Cu}(\mathrm{OH})_{2 \text { (superficie) }} \rightarrow \mathrm{Zn}_{n-x} \mathrm{Cu} \mathrm{S}_{n} \cdot x \mathrm{Zn}(\mathrm{OH})_{2 \text { (superficie) }}
$$

El cobre incorporado a la red cristalina de la esfalerita se reducirá oxidando al sulfuro $\left(\mathrm{S}^{2-}\right)$, para producir una especie rica en azufre $\left(\mathrm{CuS}+\mathrm{Cu}_{2} \mathrm{~S}+\mathrm{S}^{\circ}\right)$, de naturaleza hidrofóbica, como se discutió anteriormente.

\subsubsection{Efecto del $\mathrm{pH}$ sobre la hidrofobicidad de la esfalerita activada con $\mathrm{Cu}^{2+}$}

El valor de $\mathrm{pH}$ al cual se lleve a cabo el acondicionamiento y flotación del mineral es una variable importante ya que, como se muestra en la figura 5, las propiedades hidrofóbicas del mineral originalmente acondicionado a $\mathrm{pH}$ natural $(5,4)$, se incrementan a medida que el $\mathrm{pH}$ "de flotación" se incrementa de 5 a 9 , lo cual se debe a que en el rango ácido el ion $\mathrm{H}^{+}$, dada su mayor actividad, interactúa con las especies de azufre (e.g., $\mathrm{S}^{2-}, \mathrm{S}^{\circ}$ y $\mathrm{S}_{\mathrm{n}}{ }^{2-}$ ), generando sitios hidrofílicos del tipo HS. Este papel de los protones 
ESTUDIO DE LOS MECANISMOS DE ACTIVACIÓN DE LA ESFALERITA CON CU(II) Y PB(II) CONTACT ANGLE STUDY ON THE ACTIVATION MECHANISMS OF SPHALERITE WITH CU (II) AND PB (II)

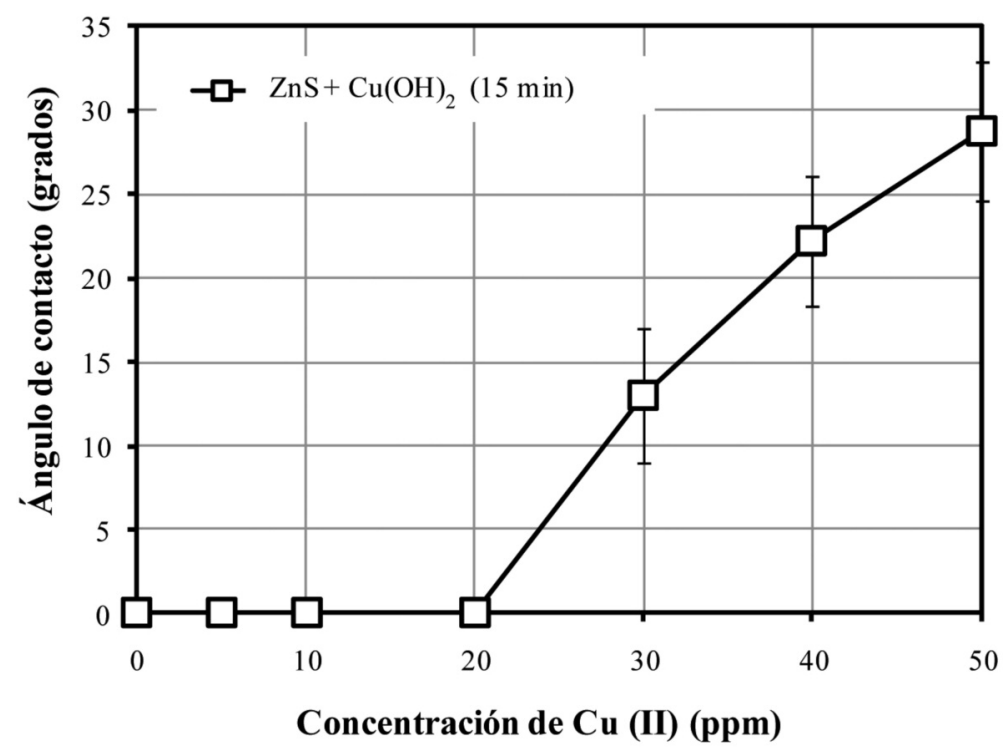

Figura 4. Efecto de la concentración de $\mathrm{Cu}$ (II) en forma de $\mathrm{Cu}(\mathrm{OH})_{2}$ sobre el ángulo de contacto de la esfalerita para diferentes tiempos de acondicionamiento. Las pruebas se realizaron a $30^{\circ} \mathrm{C}, \mathrm{pH} 9$ y una fuerza iónica de $10^{-3} \mathrm{M}$ de $\mathrm{NaNO}_{3}$.

Figure 4. Effect of the concentration of $\mathrm{Cu}(\mathrm{II})$ as $\mathrm{Cu}(\mathrm{OH})_{2}$ on the contact angle of sphalerite at different conditioning times. The tests were performed at $30^{\circ} \mathrm{C}, \mathrm{pH} 9$ and ionic strength of $10^{-3} \mathrm{M} \mathrm{NaNO}_{3}$.

se hace menos significativo a medida que la solución se torna neutra o ligeramente alcalina; sin embargo, para valores de $\mathrm{pH}$ por arriba de 9 , los iones $\mathrm{OH}^{-}$, que han incrementado su actividad, interactúan a su vez con los sitios metálicos de la superficie (e.g., $\mathrm{Cu}^{1+} \mathrm{y}$ $\mathrm{Zn}^{2+}$ ), lo que finalmente también se refleja en un incremento de sitios hidrofílicos y en una disminución del ángulo de contacto y, por lo tanto, de la flotabilidad del mineral. Como se observa en la figura 5, parece ser que a un $\mathrm{pH}$ de alrededor de 9 , el ángulo de contacto que la esfalerita desarrolla debido a la activación con $\mathrm{Cu}(\mathrm{II})$ alcanza su valor máximo $\left(36^{\circ}\right)$.

\subsubsection{Efecto de la interacción del xantato con la esfalerita activada con cobre}

La activación de esfalerita con $\mathrm{Cu}$ (II) da lugar a la formación de una capa conductora de calcocita $\left(\mathrm{Cu}_{2} \mathrm{~S}\right)$, mientras que la esfalerita es naturalmente aislante, permitiendo así que los colectores tipo tiol (e.g., xantato isopropílico de sodio) formen complejos insolubles del tipo CuX en la superficie de la esfalerita, de acuerdo a reacciones como la siguiente ${ }^{[7 \text { y } 9]}$ :

$$
\mathrm{Cu}_{2} \mathrm{~S}+\mathrm{X}^{-} \rightarrow \mathrm{CuX}+\mathrm{CuS}^{-}
$$

Con la intención de evaluar el efecto del acondicionamiento con xantato $\left(10^{-4} \mathrm{M} \mathrm{XINa}, \mathrm{pH} 9\right)$, de la esfalerita activada con $\mathrm{Cu}^{2+}(\mathrm{pH} 5,4)$ y $\mathrm{Cu}(\mathrm{OH})_{2}$ $(\mathrm{pH} 9)$, sobre la hidrofobicidad del mineral, se diseñaron y realizaron experimentos cuyos resultados se presentan en la figura 6 , en la cual se observa que independientemente de la especie activadora $\left(\mathrm{Cu}^{2+}\right.$ o $\mathrm{Cu}(\mathrm{OH})_{2}$ ), el ángulo de contacto aumenta con la concentración de $\mathrm{Cu}$ y que el intercambio iónico (i.e., presencia de $\mathrm{Cu}^{2+}$ ), es más efectivo que la precipitación superficial (i.e., presencia de $\mathrm{Cu}(\mathrm{OH})_{2}$ ); por ejemplo, para una concentración de $50 \mathrm{ppm}$ de $\mathrm{Cu}^{2+}$ y $10^{-4} \mathrm{M}$ de XINa, se observa un ángulo de contacto de $62^{\circ}$, mientras que para una concentración de 50 ppm de $\mathrm{Cu}(\mathrm{II})$ en forma de $\mathrm{Cu}(\mathrm{OH})_{2}$ y la misma concentración de colector, el ángulo de contacto obtenido es de $54^{\circ}$. Este comportamiento corrobora los resultados obtenidos en las figuras 3 y 4 , en las que se observa claramente que el mecanismo de intercambio iónico presenta una mayor eficiencia en comparación con el mecanismo de activación por $\mathrm{Cu}(\mathrm{OH})_{2}$, debido a que éste último necesita un 


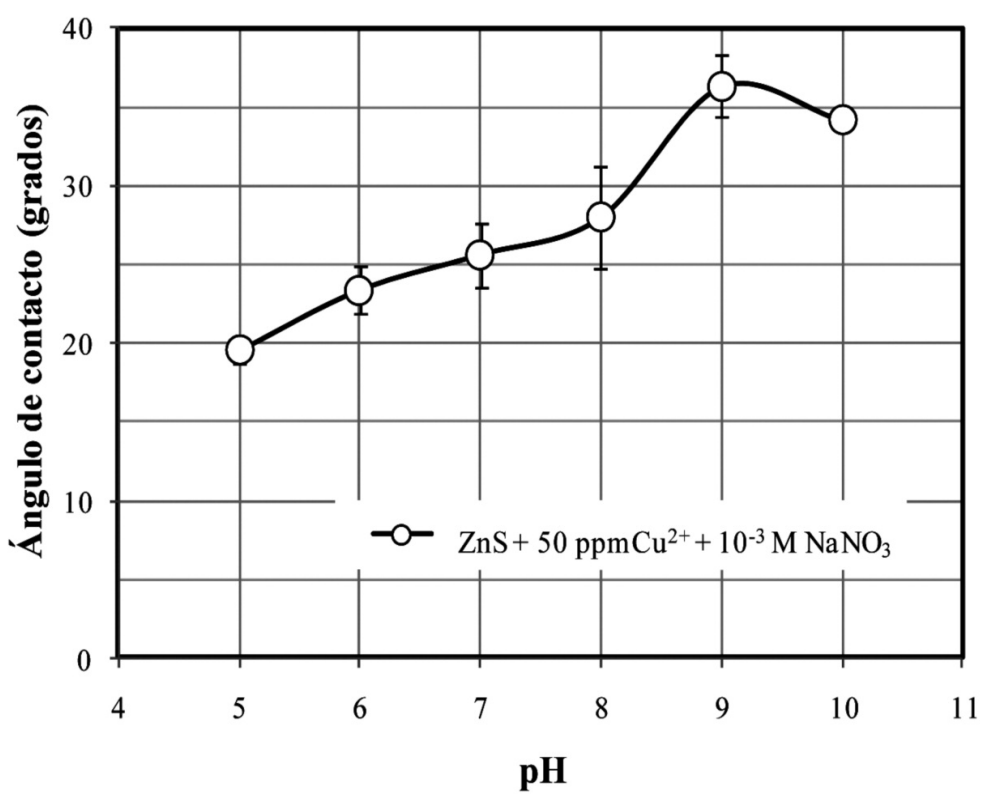

Figura 5. Efecto del $\mathrm{pH}$ sobre la hidrofobicidad inducida por la activacion con $\mathrm{Cu}^{2+}(\mathrm{HCl}$ y $\mathrm{NaOH} 0,1 \mathrm{M})$. Los experimentos se realizaron a $30^{\circ} \mathrm{C}$ y $10^{-3} \mathrm{M} \mathrm{NaNO}_{3}$.

Figure 5. Effect of $\mathrm{pH}$ on the hydrophobicity induced by activation with $\mathrm{Cu}^{2+}(\mathrm{HCl}$ and $\mathrm{NaOH} 0.1 \mathrm{M})$. The experiments were performed at $30^{\circ} \mathrm{C}$ and $10^{-3} \mathrm{M} \mathrm{NaNO}_{3}$.

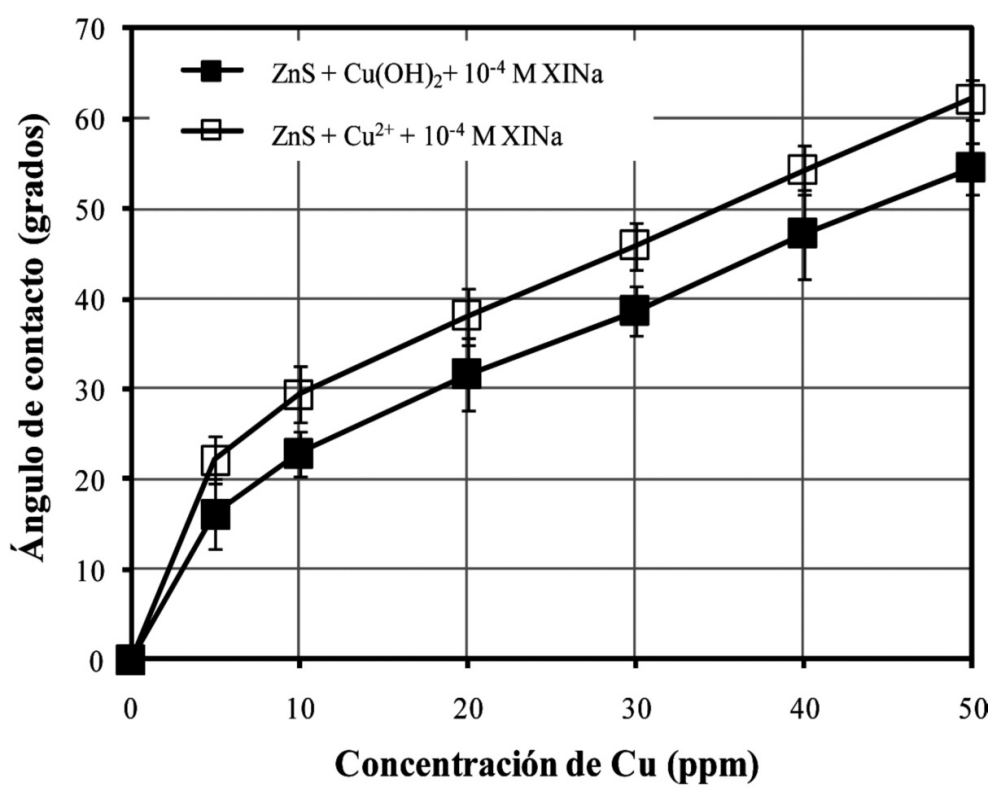

Figura 6. Efecto de la interacción entre el XINa y la esfalerita activada con distintas concentraciones de $\mathrm{Cu}^{2+}(\mathrm{pH} 5,4)$ y $\mathrm{Cu}(\mathrm{OH})_{2}(\mathrm{pH} 9)$.

Figure 6. Effect of the interaction between XINa and sphalerite activated with different concentrations of $\mathrm{Cu}^{2+}(p H$ 5.4) and $\mathrm{Cu}(\mathrm{OH})_{2}(\mathrm{pH} 9)$. 
tiempo mayor para que el $\mathrm{Cu}(\mathrm{II})$ sea adsorbido en la superficie mineral, lo que da como consecuencia menos sitios disponibles para la reacción entre el cobre y el xantato. Sin embargo, se puede decir que la diferencia observada es relativamente menor y que los resultados obtenidos con ambos acondicionamientos son comparables.

\subsection{Activación de esfalerita con $\mathrm{Pb}$ (II)}

Para evaluar la contribución del fenómeno de activación de esfalerita con $\mathrm{Pb}$ (II) bajo las diversas condiciones que prevalecen en la flotación (e.g., presencia y ausencia de oxígeno y colector), se diseñaron los experimentos descritos en la tabla II. Los resultados se muestran en la figura 7, en la que se presenta el ángulo de contacto en función de la concentración de $\mathrm{Pb}(\mathrm{II})$, observándose que independientemente de la atmósfera empleada en el acondicionamiento (i.e., aire o $\mathrm{N}_{2}$ ), el ángulo de contacto se incrementa a medida que la concentración de la especia activadora aumenta en la solución, de acuerdo al mecanismo que describe la siguiente reacción ${ }^{[10]}$ :

$$
\mathrm{ZnS}_{(\mathrm{S})}+\mathrm{Pb}(\mathrm{OH})_{2(S)} \rightarrow \mathrm{PbS}_{(\mathrm{S})}+\mathrm{Zn}(\mathrm{OH})_{2(\mathrm{~S})}
$$

Este mecanismo ocurre de manera natural debido a que la solubilidad del producto $\mathrm{PbS}$ es menor que la solubilidad de la esfalerita. A diferencia del cobre, el plomo no sustituye al zinc de la red del cristal, sino que se precipita sobre su superficie como producto de la reacción con el ión sulfuro $\left(\mathrm{S}^{2-}\right)$ que resulta de la solubilidad, aunque pequeña $\left(\mathrm{K}_{\mathrm{ps}}=10^{-25}\right.$, comparada con $\mathrm{K}_{\mathrm{sp}}=10^{-28}$ para la galena), de la esfalerita.

La figura 7 también muestra que el oxígeno desempeña un papel importante durante la activación, ya que puede inclusive promover la flotabilidad natural de la esfalerita. Como se observa para 200 ppm de $\mathrm{Pb}(\mathrm{II})$ en ausencia de xantato, en atmósferas de nitrógeno (i.e., baja concentración de oxígeno disuelto), se obtiene un ángulo de contacto de alrededor de $12,6^{\circ}$, el cual se incrementa en atmósferas de aire debido a la presencia de oxígeno hasta alcanzar un valor de $23^{\circ}$. El mismo comportamiento se observa con la esfalerita activada con $\mathrm{Pb}(\mathrm{II})$ y acondicionada con XINa, donde se obtienen ángulos de $37^{\circ}$ y $51^{\circ}$, respectivamente, lo que evidencia el efecto benéfico de la presencia de oxígeno. El mayor ángulo de contacto observado en atmósferas de aire respecto al observado en atmósferas de nitrógeno, se puede explicar, de acuerdo con Trahar y col.[10], en términos del papel catalizador que el $\mathrm{Pb}^{2+}$ pudiera jugar en la reacción de oxidación del mineral para

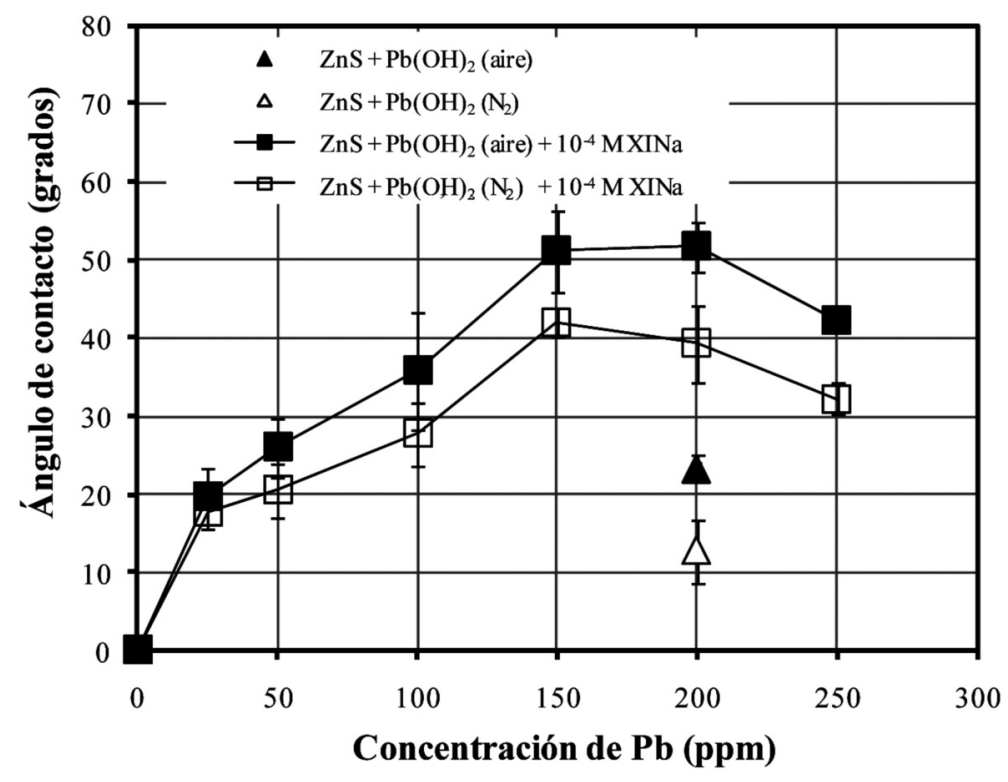

Figura 7. Efecto de la concentración de $\mathrm{Pb}$ (II) sobre el ángulo de contacto de la esfalerita activada bajo atmósferas de aire y nitrógeno, y posteriormente acondicionada con $10^{-4} \mathrm{M}$ de $\mathrm{XINa}$ a $\mathrm{pH} 9$.

Figure 7. Effect of the concentration of the $\mathrm{Pb}$ (II) on the contact angle of sphalerite activated under air and nitrogen atmospheres and then conditioned with $10^{-4} \mathrm{M}$ XINa at $\mathrm{pH} 9$. 
producir azufre elemental, la cual ocurre según la siguiente reacción:

$$
\mathrm{ZnS}_{(s)}+\frac{1}{2} \mathrm{O}_{2}+\mathrm{H}_{2} \mathrm{O} \stackrel{\mathrm{Pb}^{4+}}{\longrightarrow} \mathrm{Zn}_{(\mathrm{ac})}^{2+}+\mathrm{S}_{(s)}+2 \mathrm{OH}_{(a c)}^{-}
$$

La presencia de azufre elemental sobre la superficie de la esfalerita activada con $\mathrm{Pb}(\mathrm{II})$ coadyuva a la hidrofobicidad producida por la adsorción de xantato, lo que se ve reflejado en una mayor hidrofobización, es decir, en la obtención de mayores ángulos de contacto.

\subsubsection{Efecto del pH sobre la activación de la esfalerita con $\mathrm{Pb}(\mathrm{II})$}

$\mathrm{El} \mathrm{pH}$ de acondicionamiento es muy importante ya que de éste depende la especie predominante en el sistema acuoso de interés. La figura 8 presenta el diagrama de distribución de especies de plomo ${ }^{[11]}$, en el que se observa que el dominio del catión $\mathrm{Pb}^{2+}$ se localiza a valores de $\mathrm{pH}$ menores de 5,7 y que estos precipitan como hidróxidos metálicos a $\mathrm{pH}$ alcalino, mientras que en la transición de $\mathrm{pH}$ neutro a alcalino se da la formación de diversos complejos hidroxo, en particular el $\mathrm{PbOH}^{+}$, especie con gran afinidad por interfaces mineral-solución acuosa. Lo anterior indica que dependiendo del $\mathrm{pH}$ de acondicionamiento, pueden ocurrir varias reacciones entre las especies metálicas en solución y la superficie mineral.

Los resultados obtenidos se muestran en la figura 9, en la que se presenta el efecto del $\mathrm{pH}$ de acondicionamiento sobre el ángulo de contacto, el cual se incrementa de manera significativa al aumentar el $\mathrm{pH}$, hasta alcanzar un valor máximo a $\mathrm{pH} 8$, que se mantiene a $\mathrm{pH}$ 9. Este comportamiento se debe a que valores de $\mathrm{pH}$ moderadamente alcalinos favorecen la adsorción de $\mathrm{PbOH}^{+}$, especie altamente surfactante, sobre la superficie de la esfalerita, mientras que a valores de $\mathrm{pH}$ menores a 7 la especie predominante es el catión $\mathrm{Pb}^{2+}$, quien no posee la afinidad por la interfase que posee el complejo hidroxo; como consecuencia, la actividad del $\mathrm{PbOH}^{+}$en la interfase agua-mineral, es mayor que la actividad del catión $\mathrm{Pb}^{2+}$, lo que favorece la precipitación de $\mathrm{PbS}$ en el primer caso.

\section{CONCLUSIONES}

En base a los resultados presentados y discutidos en este trabajo, se obtuvieron a las siguientes conclusiones.

- La hidrofobización de la esfalerita debido a la activación con $\mathrm{Cu}$ (II) se incrementa con la concen-

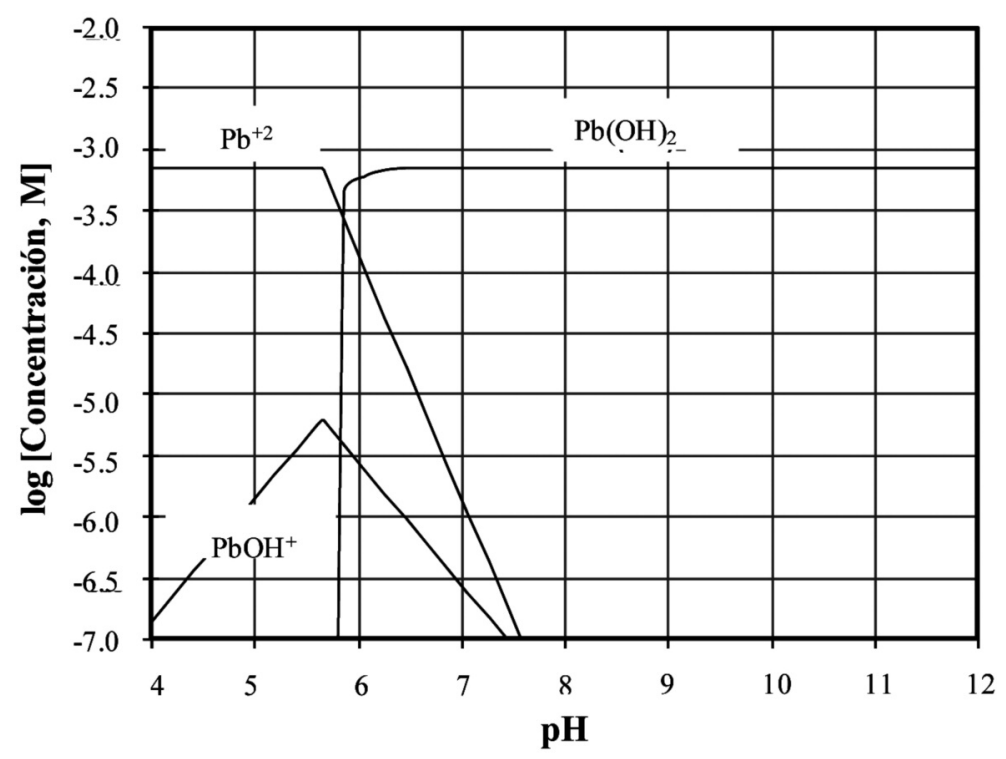

Figura 8. Diagrama de distribución de especies del sistema $\mathrm{Pb}^{2+}-\mathrm{H}_{2} \mathrm{O}$ a $25^{\circ} \mathrm{C}$ y una concentración inicial de $\mathrm{Pb}^{2+}$ de $7,23 \times 10^{-4} \mathrm{M}(150 \mathrm{ppm})^{[10]}$.

Figure 8. Species distribution diagram of the $\mathrm{Pb}-\mathrm{H}_{2} \mathrm{O}$ system at $25^{\circ} \mathrm{C}$ and $\mathrm{Pb}^{2+}$ initial concentration of $7.23 \times 10^{-4}$ $M(150 \mathrm{ppm})^{[10]}$. 


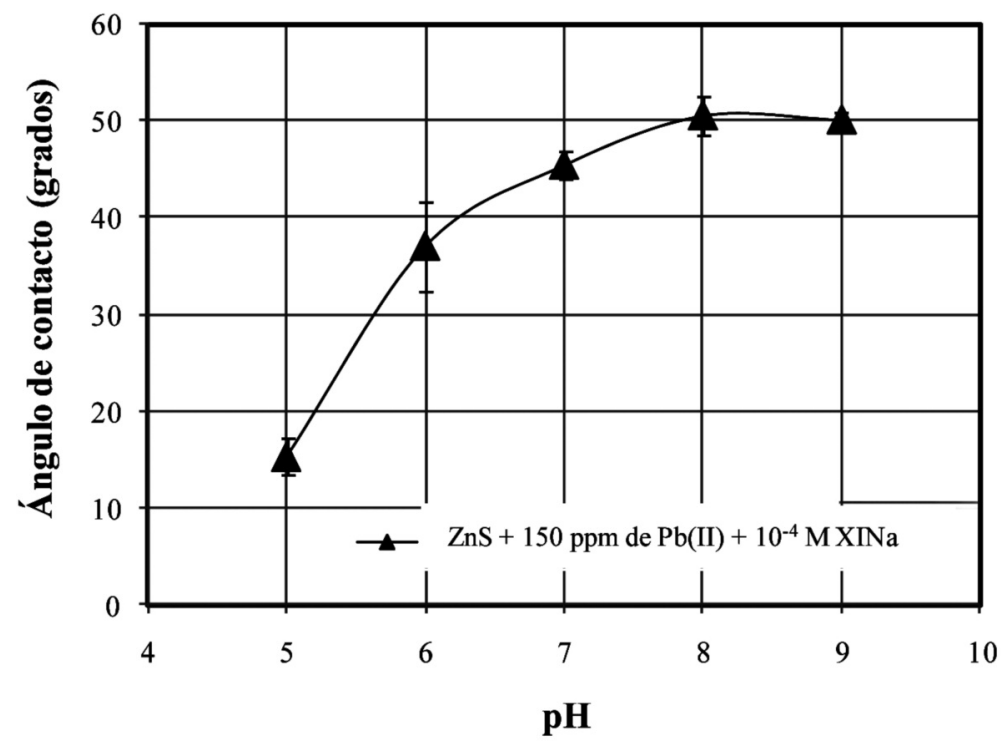

Figura 9. Efecto del pH sobre el ángulo de contacto de la esfalerita que resulta de la activación con $\mathrm{Pb}(\mathrm{II})$ y su respuesta a la presencia de xantato $(\mathrm{pH}=9)$.

Figure 9. Effect of $\mathrm{pH}$ on the contact angle of sphalerite resulting from the activation with $\mathrm{Pb}(\mathrm{II})$ and its response to the presence of xanthate $(\mathrm{pH}=9)$.

tración de cobre, con el tiempo de acondicionamiento y se favorece cuando se efectúa a $\mathrm{pH}$ natural $(5,4)$, debido a la mayor actividad de la especie $\mathrm{Cu}^{2+}$ que se adsorbe sobre la esfalerita y se intercambia por los iones zinc de las capas exteriores del mineral, en comparación con la activación a $\mathrm{pH}$ 9, en el que la especie predominante es el $\mathrm{Cu}(\mathrm{OH})_{2}$.

- El xantato se quimisorbe en el sitio cuproso de la superficie de la esfalerita activada incrementándole su hidrofobicidad, independientemente del mecanismo de activación (i.e., intercambio iónico y precipitación superficial), por lo que se puede concluir que ambos mecanismos producen resultados equiparables.

- En el caso de la activación de la esfalerita con $\mathrm{Pb}(\mathrm{II})$, la presencia de oxígeno promueve el desarrollo de propiedades hidrofóbicas debido a que la presencia de $\mathrm{Pb}$ (II) favorece la reacción de oxidación del $\mathrm{S}^{2-}$ de la esfalerita con el oxígeno disuelto para producir $\mathrm{S}^{\circ}$.

- La hidrofobización de la esfalerita causada por la activación con $\mathrm{Pb}(\mathrm{II})$ se incrementa con la concentración de $\mathrm{Pb}(\mathrm{II})$ (hasta 150 ppm), y se ve favorecida a valores de $\mathrm{pH}$ neutros y alcalinos, debido a la adsorción de especies con alta afinidad por las interfases, como son el complejo hidroxo $\mathrm{PbOH}^{+}$y el hidróxido de plomo.
- Para los tiempos de acondicionamiento y concentraciones de $\mathrm{Pb}$ (II) empleados, el ángulo de contacto desarrollado por la esfalerita activada con $\mathrm{Pb}(\mathrm{II})$ en atmósferas de aire y en presencia de xantato isopropílico, puede ser del mismo orden de magnitud (ca. $50^{\circ}$ ), que cuando la esfalerita se activa deliberadamente para flotarla con xantato, lo que representa un reto de optimización de la flotación selectiva plomo/zinc.

\section{Agradecimientos}

Los autores agradecen al CONACYT el financiamiento recibido para la realización del presente estudio. También agradecen el apoyo del personal técnico y administrativo de la Unidad Saltillo del CINVESTAV-IPN, y el apoyo del M.C. Juan Antonio González Anaya, responsable del Laboratorio de Metalurgia Extractiva.

\section{REFERENCIAS}

[1] P.J. Guy y W.J. Trahar, Flotation of Sulphide Minerals, Developments in Mineral Processing 6, Ed. Forssberg, Amsterdam, 1985, pp. 91-110. 
[2] M.C. Fuerstenau y P. Somasundaran, Flotation. En Principles of Mineral Processing (M.C. Fuerstenau y K.N. Han, Eds.). SME, EE.UU., 2003, pp. 245-306.

[3] T.T. Chau, Miner. Eng., 22 (2009) 213-219.

[4] P.T.L. Koh y M.P. Schwarz, Miner. Eng., 19 (2006) 619-626.

[5] I.W. Wark y A.B. Cox, Transactions of the American Institute of Mining and Metallurgical Engineers 112 (1934) 189-244.

[6] N. P. Finkelstein, Int. J. Miner. Process. 52 (1997) pp. 81-120.
[7] A.P. Chandra y A.R. Gerson, Adv. Colloid. Interfac. Sci. 145 (2009) 97-110.

[8] C.A. Prestidge, W.M. Skinner, J. Ralston y R.St.C. Smart, Appl. Surf. Sci. 108 (1997) 333-344.

[9] A.C.P. Duarte y S.R. Grano, Miner. Eng., 20 (2007) 766-775.

[10] W.J. Trahar, G.D. Senior, G.W. Heyes y M.D. Creed, Int. J. Miner. Process. 49 (1997) 121-148.

[11] E.T. Pecina, Tesis Doctoral, Centro de Investigación y de Estudios Avanzados del Instituto Politécnico Nacional, Unidad Saltillo, México 2003. 